history of decreased vision and bilateral floaters. Visual acuity was $6 / 18-2$ in the left eye and $6 / 60$ in the right. Dilated fundus examination showed bilateral vitreous haemorrhages, with neovascularisation at the disc and elsewhere, and tractional retinal detachments. Non-fasting pinprick blood glucose was $20.3 \mathrm{mmol} / \mathrm{L}$ and blood pressure was 140/100 $\mathrm{mmHg}$.

The patient denied weight loss (BMI $18 \mathrm{~kg} / \mathrm{m}^{2}$ ) or polydipsia, although detailed questioning revealed a 20-year history of approximately 5 litres of fluid intake daily, predominantly sugary drinks. Three years before, he experienced slurred speech and pins and needles in his right arm, which was attributed to a viral infection and no investigations were done.

He was diagnosed with type 2 diabetes mellitus (negative anti-GAD antibodies and anti-IA-2 antibodies). Oral hypoglycaemics were commenced. He underwent urgent bilateral pan-retinal photocoagulation, then sequential vitrectomies with membrane dissection, endolaser, and gas tamponade. Visual acuity at the last clinic visit was $6 / 9$ in the left and $6 / 36$ in the right, attributable to diabetic macular oedema.

This unusual case highlights the importance of investigating neurovascular signs and symptoms, to include blood glucose measurement, as diabetes is increasingly seen in young working-age patients who neither report their longstanding diabetic symptoms nor conform to the metabolic syndrome'. The potential morbidity and mortality impact of undiagnosed diabetes underscores the importance of campaigns focusing on improving patient awareness of diabetic symptoms.

Jacintha Gong,

Ophthalmology Specialty Registrar, NHS

Tayside.

Email: jacinthagonglanhs.net

Thomas GM Cormack,

Consultant Ophthalmologist and Lead Clinician, Department of Ophthalmology, NHS Tayside.

\section{Patient consent}

The patient has consented to publication of this letter.

\section{REFERENCE}

1. Symes RJ, Liew G, Tufail A. Sight-threatening diabetic eye disease: an update and review of the literature. Br J Gen Pract 2014; DOI: 10.3399/ bjgp14X682033.

\section{Non-contact infrared thermometers}

I wrote a letter to the $B M J$ last year setting out the evidence for non-contact infrared thermometry in adults. ${ }^{1}$ Unlike paediatric use, ${ }^{2}$ my conclusion was that these devices are not reliable in adults, largely because vasoconstriction of the blood supply to their foreheads and perspiration leading to a dangerously high false negative rate. A similar conclusion has been reached by the Scottish Health Technology Group, advising against their use for adults in Scottish NHS hospitals. $^{3}$

\section{Gervase Vernon,}

GP, John Tasker House Surgery.

E-mail: gvernondanhs.net

\section{REFERENCES}

1. Vernon $G$. Inaccuracy of forehead thermometers (letter). BMJ 2013; 346: f1747.

2. Wang $K$, Gill $P$. Wolstenholme J, et al. Noncontact infrared thermometers for measuring temperature in children: primary care diagnostic technology update. Br J Gen Pract 2014; DOI: 10.3399/bjgp14X682045

3. Marsden A. Re: Inaccuracy of forehead thermometers (electronic letter). BMJ 2013; 346:

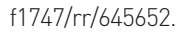

DOI: 10.3399/bjgp14X682669

\section{Headaches in the absence of other signs do not require imaging by GPs}

Taylor et al provide an interesting review on the timeless conundrum of headaches and brain tumours. ${ }^{1}$ Among the discussion they seem to advocate easier access to brain imaging in primary care. This is a shame, especially since the question was answered by a well-written study (by two of the same authors) in the BJGP 7 years ago. ${ }^{2}$ I use that paper to teach medical students and GP trainees that the positive predictive value of a headache, for a brain tumour, is $0.09 \%$. (In other words, if you have a headache there's only a $0.09 \%$ chance of it being a brain tumour). A new onset seizure, on the other hand, has a PPV of $1.2 \%$ for a brain tumour. The problem with imaging people's brains 'just to make sure' is, as the authors rightly point out, an incidental finding. A well carried out study showed $0.47 \%$ of healthy young men have an intracranial tumour, ${ }^{3}$ which is slightly more than the positive predictive value of a headache anyway. Taylor et al also, erroneously, remark the commonest symptom' of a brain tumour is headache, but in fact only $10 \%$ of people with a brain tumour ever report a headache before the diagnosis. Easier access to brain imaging for GPs will only mean one thing: more brain scans. And more brain scans means only one thing: more incidental findings.

Oliver Dominic Starr,

Sessional GP, Hertfordshire.

E-mail: oliverstarrahotmail.com

\section{Competing interests}

The author missed a brain tumour 3 years ago in a young woman presenting with headaches (the patient is now well). He has seen a similar patient have a meningioma, erroneously attributed to the patient's headaches, resected; the headaches persisted despite the surgery and abated once the patient's emotional state had improved.

\section{REFERENCES}

1. Taylor T, Evangelou N, Porter H, et al. Headache: two views on the right approach in general practice. Br J Gen Pract 2014; 64(626): 475-476.

2. Hamilton W, Kernick D. Clinical features of primary brain tumours: a case control study using electronic primary care records. Br J Gen Pract 2007; 57(542): 695-699.

3. Weber F, Knopf H. Incidental findings in magnetic resonance imaging of the brains of healthy young men. J Neurological Sci 2006; 240: 81-84.

DOI: 10.3399/bjgp14X682681

\section{Family and Friends Test}

The NHS Family and Friends Test (FFT) will be implemented into general practice in December $2014^{1}$ and The NHS Strategic Projects Team is supporting this implementation across the Midlands and East region.

In our workshops, GPs and managers initially considered FFT another mechanism for complaints' generation; but training has demonstrated that FFT can promote a better relationship between 
patients and practices. Our pilot sites have report being pleasantly surprised at the feedback received from patients. This has been overwhelmingly positive, expressing gratitude to the practice for its quality of care; for the first time, a technique is being introduced into general practice which will produce consistent, monthly feedback of the patient experience. Any issues have usually been relatively minor in nature requiring small adjustments to service delivery.

NHS England has produced guidance for general practice. ${ }^{2}$ Every practice will ask the same initial question: how likely are you to recommend our GP practice to friends and family if they needed similar treatment or care? Patients have the opportunity of scoring this question as very likely; likely; no more likely than unlikely; unlikely; very unlikely; or don't know. The results are submitted to NHS England monthly. How the score will be calculated will be based on further guidance from NHS England. A second question, which can be of the practice's choosing, asks for further information and can target specific local issues if required.

FFT has been used in industry for many years; it is only new to the NHS. Hospitals have used the feedback to adjust services reflecting patient commentary. Examples can be found on our website: http:// www.thestrategicprojectsteam.co.uk/. The information will assist colleagues to implement the Friends and Family Test and use it to improve the patient experience further.

\section{Eric Saunderson,}

GP Lead, NHS Strategic Projects Team.

E-mail: eric.saundersonanhs.net

\section{REFERENCES}

1. General Practitioners Committee, NHS England, NHS Employers. General Medical Services Contract 2014/15: Guidance and Audit Requirements, NHS Gateway reference: 01347. Leeds: NHS Employers, 2014

2. NHS England. The Friends and Family Test in General Practice Guidance, NHS England Gateway reference: 01788. Redditch, NHS England, 2014.

DOI: 10.3399/bjgp14X682693

\section{Young carers}

The editorial on child maltreatment in the
September issue of the BJGP prompted me to think again about young carers. ${ }^{1}$ It talked about the fact that many maltreated and vulnerable children do not have an administratively competent carer and that often parents have health issues such as alcohol misuse or physical or mental health problems that can affect their children. In general practice it is not uncommon for us to see the parents but not see the young people themselves. However, young carers are at risk of social isolation and bullying, under-achievement, absenteeism from school, and physical and mental ill health. Having a holistic approach to family medicine should include supporting the young people in their caring role. We can do this by explaining to parents we are happy to support their children too. We can also signpost them to useful websites like www.youngcarers.net or local groups for young carers that can provide youth worker support, youth clubs and days out depending on the area. Perhaps I can set a challenge to GP's reading this and suggest a way to double their CPD points? Find out what is available in your area to support young carers and next time you see a patient with health issues who has children at home think also about what support their children may need.

\section{Sharmila Parks,}

GP Talbot Medical Centre, South Tyneside and Member of the RCGP Adolescent Health Group.

E-mail: sharmila.parkslanhs.net

\section{REFERENCE}

1. Woodman J, Rafi I, de Lusignan S. Child maltreatment: time to rethink the role of general practice. Br J Gen Pract 2014; 64(626): 444-445.

\section{Should we celebrate outstanding achievement in general practice training?}

Celebrating achievement aims to boost morale and inspire others. At a time when general practice is under intense pressure, we on the London GP Trainee Committee thought it was important to do just that and we organised the first London Trainee Excellence awards, based on a model initiated by the Severn Deanery.

In his letter in the October edition of this journal, ${ }^{1}$ Dr Spitzer questions whether we can define excellence in general practice, should we celebrate it and if patients would be better off if trainees focused on 'core general practice' and covering the curriculum rather than activities outside the traditional model of the doctor alleviating the suffering of their individual patients.

We too struggled with defining excellence. We did not include clinical proficiency in our criteria as we felt this is reflected in attainment of MRCGP and we were seeking to focus on the broader meaning of general practice as it has become, with a population as well as individual focus. Research (as reflected in this journal), teaching, and leadership are all important aspects of professional development, being a GP, and core general practice. Indeed this is reflected in the RCGP curriculum and competencies.

The categories we chose; Research, Learning and Development, Leadership, Medical Work outside the vocational training scheme, and Personal Achievement outside Medicine, allowed trainers and trainees to nominate their colleagues for a range of awards and to showcase the many opportunities available to trainee GPs. The award winners are all hardworking, dedicated doctors, who committed much of their personal time to their projects and I am sure they would be dismayed if they were seen to be neglecting their clinical duties.

Taking part in activities outside direct patient care should not negatively impact on clinical ability. Quite the opposite, it can help with personal and professional development, building the skills to be the adaptable, innovative workforce required to maintain one of the most effective primary care systems in the world.

Celebrating outstanding achievements inspires others and provides positive feedback, improving resilience in these challenging times.

Bridget Kiely,

Darzi Fellow in Clinical Leadership, Health Education South London.

E-mail: bridget.kielylasouthlondon.hee.nhs.uk

Swati Sheth, Shreya Dhar,

Anjella Balendra and Anna McGlone,

Health Education South London. 